\title{
Gate leakage suppression and contact engineering in nitride heterostructures
}

\author{
Yuh-Renn Wu, ${ }^{\text {a) }}$ Madhusudan Singh, and Jasprit Singh \\ Department of Electrical Engineering and Computer Science, University of Michigan, \\ Ann Arbor, Michigan 48109
}

(Received 10 June 2003; accepted 25 August 2003)

\begin{abstract}
We present a self-consistent approach to examine current flow in a general metal-polar heterostructure junction. The approach is applied to examine properties of three classes of junctions that are important in devices: (i) GaN/AlGaN structures that are used in nitride heterojunction field effect transistors; (ii) GaN/AlGaN/high- $\kappa$ insulator structures for potential application in very small gate devices to suppress gate tunneling current; and (iii) GaN/AlGaN/polar insulator junctions with practical application for low source resistance regions. The physical parameters used for high- $\kappa$ dielectrics and polarization charges reflect values typically found in ferroelectric materials. Our studies indicate that tailoring of junction properties is possible if a dielectric thicknesses of $\sim 20 \AA$ can be achieved. (C) 2003 American Institute of Physics. [DOI: 10.1063/1.1618926]
\end{abstract}

\section{INTRODUCTION}

GaN/AlGaN heterostructure field effect transistors (HFETs) have attracted considerable interest owing to their potential use in high power, high-voltage, and hightemperature electronic and optoelectronic devices. ${ }^{1-5}$ In particular, it is known that there is a large spontaneous polarization in the nitrides. ${ }^{6,7}$ Additionally, the piezoelectric effect is also very strong. As a result, there is a strong fixed polarization charge at the heterointerface, ${ }^{8,9}$ which can introduce very large electric fields $\left(\sim 10^{6} \mathrm{~V} \mathrm{~cm}^{-1}\right)$ and band bending, and induce a two-dimensional electron gas (2DEG) $\left(\sim 10^{13} \mathrm{~cm}^{-2}\right)$ in the heterostructure interface without intentional doping. ${ }^{10}$ For certain designs, a two-dimensional hole gas (2DHG) can also be induced.

Advances in nitride heterostructure technology have attracted careful studies of junction properties of metal-polar heterostructure combinations where dopants are replaced by built-in polar fixed charges. Very large band bending caused by interface fixed charges permits the tailoring of the current-voltage $(I-V)$ relations ${ }^{11}$ by simply controlling the $\mathrm{AlGaN}$ layer thickness. It is important to develop a theoretical model that can predict the $I-V$ relationship in a general metal-polar heterostructure system. In this article, we will present such a model and apply it to three classes of junctions that are important for device technologies: (i) GaN/ $\mathrm{AlGaN}$ junctions, especially when the AlGaN (high band gap) layer is thin so that tunneling current becomes significant. This study is important to understand gate current in very small $(\leqslant 0.25 \mu \mathrm{m})$ HFETs; (ii) GaN/AlGaN/high- $\kappa$ dielectric insulator junctions. We use typical values for material parameters found in materials, such as $\mathrm{LiNbO}_{3}$, to examine if such junctions can reduce gate tunneling without significantly influencing gate control of the channel; and (iii) GaN/AlGaN/polar dielectric junctions for application in very

a)Electronic mail: yrw@umich.edu low resistance junctions. Such junctions could be useful as tunneling junctions or as junctions for very low source resistance in HFETs. Experimental work in the last two classes of junctions has only begun and our theoretical studies could shed light on the kinds of structures that can have interesting properties.

In our previous work, ${ }^{11}$ we demonstrated how the polar charge and the resultant band bending can affect tunneling probabilities and $I-V$ relations in a metal heterostructure junction. In order to apply that model to more involved cases, we need to determine the current flow inside the entire device, which can be expressed by the drift-diffusion current model, as well as effects arising out of hysteresis exhibited by ferroelectric materials. ${ }^{12}$ The drift-diffusion current model has been used in numerical simulations for many decades. ${ }^{13-15}$ In most simulations, the metallurgical junction is assumed to be a continuous function of position-an assumption that is not valid for the abrupt changes encountered in the band profile of a heterojunction. Use of thermionic emission current at the heterointerface as a boundary condition is an approximation used by much of the early work. ${ }^{14} \mathrm{~A}$ more accurate model, considering tunneling and thermionic emission current for the boundary condition, has been used in more recent work. ${ }^{15}$ This model has been applied to the study of GaAs/AlGaAs high electron mobility transistors (HEMTs) and heterojunction bipolar transistors.

In this work, the tunneling current is found to be the dominant contribution to the current at the heterointerface. The drift-diffusion current model is then included in our charge control model to simulate the current flow in bulk region. It addresses charge control as well as transport through tunneling and drift diffusion. With this model, we examine the $I-V$ characteristics and sheet charge density. In Sec. II, we will discuss the formalism that constitutes the theoretical basis of this work. Results are presented in Sec. III. We conclude in Sec. IV. 


\section{FORMALISM}

In this section, we provide the formalism for the driftdiffusion charge control model. To develop the model, we need several steps:

(1) Solution of Poisson and Schrödinger equations to get the band profiles, ${ }^{10,16}$

(2) Use of the drift-diffusion current model to obtain quasiFermi levels, and

(3) Use of tunneling current as the boundary condition for the drift-diffusion current model.

The quasi-Fermi levels are then fed back into Poisson equation until the solution goes to convergence.

Poisson equation in one dimension can be written as

$$
\frac{d^{2} E_{c}(z)}{d z^{2}}=\frac{-\rho(z)}{\epsilon(z)}
$$

where $E_{c}$ is the conduction-band profile, $\rho$ is the total charge density, and $\epsilon(z)$ is the dielectric constant in the different regions of the device. The total charge density $\rho$ includes the doping charge, free charge of the holes and the electrons, and quantum confined charge:

$$
\begin{aligned}
\rho(z)= & q\left[N_{D}-N_{A}+n_{\mathrm{free}}-p_{\mathrm{free}}-\sum_{i=1}^{N_{2 \mathrm{DEG}}} N_{i} \psi_{i}^{*}(z) \psi_{i}(z)\right. \\
& \left.+\sum_{i=1}^{N_{2 \mathrm{DHG}}} P_{i} \psi_{i}^{*}(z) \psi_{i}(z)\right],
\end{aligned}
$$

where $N_{D}$ and $N_{A}$ are the effective doping concentrations, $n_{\text {free }}$ and $p_{\text {free }}$ are the electron and hole free carrier concentrations, and $N_{2 \mathrm{DEG}}$ and $N_{2 \mathrm{DHG}}$ are the number of confined states in the two bands. $\psi_{i}(z)$ are the normalized envelope functions in which the occupations are $N_{i}$ and $P_{i}$. The 2 DEG and 2 DHG in the quantum well in Eq. (2) can be determined by solving the Schrödinger equation for the subband envelope functions and their occupations. The onedimensional Schrödinger equation can be written using the perpendicular part of the effective mass tensor as follows:

$$
\left[\frac{-\hbar^{2}}{2 m^{*}} \frac{d^{2}}{d z^{2}}+\left(E_{c}-E_{i}\right)\right] \psi=0,
$$

where $m^{*}$ is the effective mass along the longitudinal direction. Once the charges are injected into the devices, the Fermi level splits into two different quasi-Fermi levels. In order to accurately calculate the charge density inside the device, we need to use the drift-diffusion model to calculate the quasi-Fermi levels in the heterostructure. In the bulk region, excluding the heterojunction interface, the current equation can be expressed by the drift-diffusion equations:

$$
\begin{aligned}
& J_{n}=-\mu_{n} n(z) \frac{\partial E_{c}}{\partial z}+q D_{n} \frac{\partial n(z)}{\partial z}, \\
& J_{p}=\mu_{p} p(z) \frac{\partial E_{v}}{\partial z}-q D_{p} \frac{\partial p(z)}{\partial z},
\end{aligned}
$$

where $\mu_{n}$ and $\mu_{p}$ are the mobilities of the electrons and holes, and $D_{n}$ and $D_{p}$ are the diffusion coefficients of elec- trons and holes. Using the Einstein relation, ${ }^{17} D / \mu$ $=k_{B} T / q$, Eqs. (4) and (5) can be rewritten as

$$
\begin{aligned}
& J_{n}=\mu_{n} n(z) \frac{\partial E_{\mathrm{fn}}(z)}{\partial z}, \\
& J_{p}=\mu_{p} p(z) \frac{\partial E_{\mathrm{fp}}(z)}{\partial z},
\end{aligned}
$$

where $E_{\mathrm{fn}}(z)$ and $E_{\mathrm{fp}}(z)$ are quasi-Fermi levels of electrons and holes. Using the continuity equation,

$$
\begin{aligned}
& \frac{\partial J_{n}}{\partial z}=q R, \\
& \frac{\partial J_{p}}{\partial z}=-q R,
\end{aligned}
$$

where $R$ represents the Schottky-Read-Hall ${ }^{17,18}$ rate and can be expressed as

$$
R=\frac{p n-n_{i}^{2}}{\tau_{n_{0}}\left(p+n_{i}\right)+\tau_{p_{0}}\left(n+n_{i}\right)} .
$$

Here $n_{i}$ is the intrinsic carrier concentration and $\tau_{n_{0}}$ and $\tau_{p_{0}}$ are the electron and hole lifetimes at equilibrium, respectively. We use the Wentzel-Kramer-Brillouin approximation to obtain the tunneling probability of the barrier from the metal to the channel region. ${ }^{11}$

Once the tunneling current is obtained, we use it at the metal-semiconductor interface. Equations (4) and (5) are then solved for the quasi-Fermi level used in the selfconsistent loop.

\section{RESULTS}

The general structure simulated by us is shown in Fig. 1. It consists of a layer of an insulator or a generic polar material grown on a $\mathrm{GaN} / \mathrm{Al}_{0.3} \mathrm{Ga}_{0.7} \mathrm{~N}$ structure. A fixed polar charge density, $1.68 \times 10^{13} \mathrm{~cm}^{-2}$ exists at the heterointerface between $\mathrm{AlGaN}$ and GaN. The fixed charge is due to the spontaneous polarization and the piezoelectric effect. The $\mathrm{GaN}(n-)$ layer is doped at $2 \times 10^{16} \mathrm{~cm}^{-3}$, and the $\mathrm{GaN}$ $(n+)$ layer is doped at $5 \times 10^{16} \mathrm{~cm}^{-3}$. The thicknesses of both layers are $1000 \AA$.

We examine three categories of the heterostructures in these simulations:

(1) The alloy $\mathrm{Al}_{0.3} \mathrm{Ga}_{0.7} \mathrm{~N}$ constitutes the barrier layer with a $\mathrm{GaN}$ channel and substrate.

(2) A high dielectric constant insulator layer is added on top of the $\mathrm{Al}_{0.3} \mathrm{Ga}_{0.7} \mathrm{~N}$ layer.

(3) A high dielectric constant polar layer (with a charge density at $1 \times 10^{14} \mathrm{~cm}^{-2}$ ) is added on top of the $\mathrm{Al}_{0.3} \mathrm{Ga}_{0.7} \mathrm{~N}$ layer instead.

The material parameters used in this work are summarized in Table I. It is interesting to digress a little here to consider possible candidates with these kind of parameters. The dielectric constant of $\mathrm{LiNbO}_{3}$ is very close to 29. In the presence of imperfect poling, the value of spontaneous polarization charge is also quite reasonable for $\mathrm{LiNbO}_{3}$, as is 


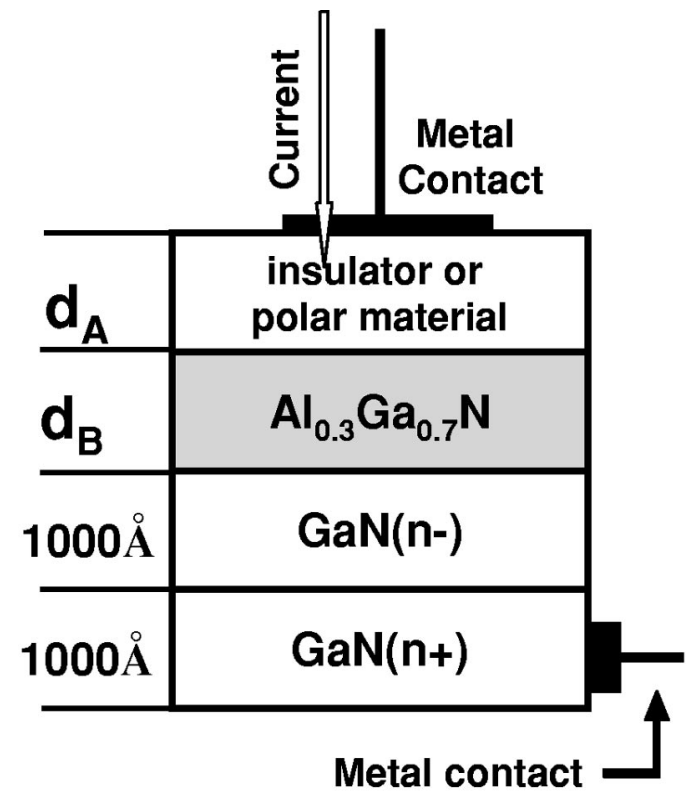

FIG. 1. A schematic of the structures considered is shown. $\mathrm{GaN}(n-)$ is doped $2 \times 10^{16} \mathrm{~cm}^{-3}$ and $\mathrm{GaN}(n+)$ substrate is doped $5 \times 10^{16} \mathrm{~cm}^{-3}$. The contact regions shown here are merely schematics, as is the rest of the figure, and do not represent any actual contact structures. The arrow represents current flow direction for positive gate bias.

the band gap. ${ }^{19,20}$ Though our calculations are not for $\mathrm{LiNbO}_{3}$ per se, its possible to use these as a guide toward designing devices with $\mathrm{LiNbO}_{3}$.

Figure 2 shows the sheet charge density and corresponding tunneling $I-V$ characteristic. We compared two barrier thickness, $50 \AA$ and $70 \AA$. As expected, the tunneling current decreases, and the sheet charge density increases with an increase in barrier thickness. In Si technology, a "rule of thumb" relating gate oxide thickness to gate length is given by

$$
L_{G} \approx 45 d_{\text {ox }} \text {. }
$$

In the nitride system, no such clear rule has been established. However, we can see from Fig. 2 that for AlGaN thickness of $\sim 50 \AA$, gate tunneling becomes very significant. It has been seen that there is a trade off between induced 2DEG charge and tunneling probability. ${ }^{11}$ For reverse biases $\approx-1 \mathrm{~V}$, the tunneling current of $70 \AA$ barrier thickness is close to that for $50 \AA$ barrier thickness. A possible reason for this could be that the increasing free carrier density cancels part of the contribution to 2DEG from a thicker barrier.

TABLE I. Material parameters for the polar and insulator layer effective masses, $m_{0}$, spontaneous polarization charge, $N_{\text {spon }}$, band gap, $E_{g}$, dielectric constant, $\kappa$ and the Schottky barrier height, $\phi_{\mathrm{SB}}$

\begin{tabular}{lcc}
\hline \hline \multicolumn{1}{c}{ Parameter } & Polar layer & Insulator layer \\
\hline$m_{0}$ & 0.2424 & 0.2424 \\
$N_{\text {spon }}\left(\mathrm{cm}^{-2}\right)$ & $1 \times 10^{14}$ & 0.0 \\
$E_{g}(\mathrm{eV})$ & 3.74 & 3.74 \\
$\kappa\left(\epsilon_{0}\right)$ & 29.0 & 29.0 \\
$\phi_{\mathrm{SB}}(\mathrm{eV})$ & 1.15 & 1.15 \\
\hline \hline
\end{tabular}
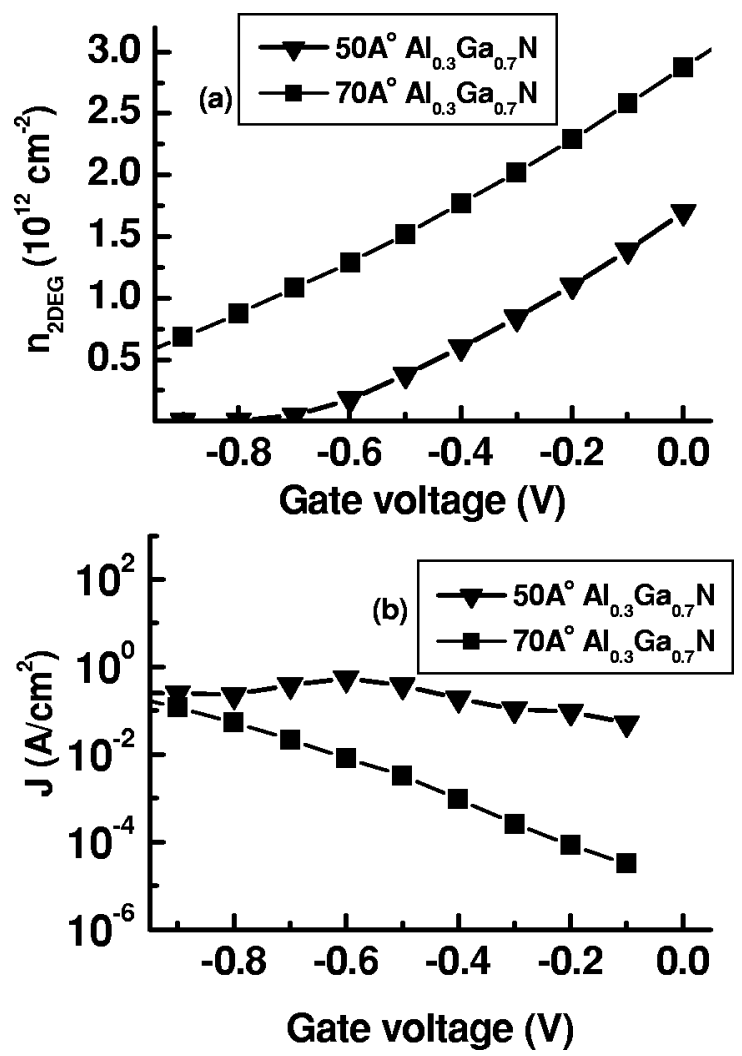

FIG. 2. The sheet charge density, $n_{2 \mathrm{DEG}}$, and $I-V$ characteristics for the device structure $\mathrm{GaN}(n+) / \mathrm{GaN}(n-) / \mathrm{Al}_{0.3} \mathrm{Ga}_{0.7} \mathrm{~N}$. Schottky barrier height, $\phi_{\mathrm{SB}}=1.45 \mathrm{eV}$.

It is well known that one way to reduce gate tunneling in field-effect transistors (FETs) is to add a high dielectric constant region between the gate and the channel. ${ }^{21,22}$ Such approaches have been demonstrated in a $\mathrm{Si}$ metal-oxidesemiconductor FET where $\mathrm{BaTiO}_{3}$ has been added. High- $\kappa$ dielectrics, such as $\mathrm{TiO}_{2}$, have been grown on nitride heterostructures. ${ }^{23}$ In Fig. 3, we show results of the calculation for structures where a material with $E_{g}=3.74 \mathrm{eV}$ and $\kappa=29 \epsilon_{0}$ has been added between the metal and AlGaN. The high- $\kappa$ material is assumed to be nonpolar. Our results indicate that 2DEG sheet charge density is relatively insensitive to an increase in the insulator thickness. However, tunneling current is reduced at least by a factor of $10^{4}$ with the introduction of an insulator layer of thickness, $d_{A}=30 \AA$. Thus, a high $-\kappa$ insulator layer can be used to decouple the relationship between barrier layer thickness and 2DEG sheet charge density.

It is also worthwhile to note that the gate control of channel properties of a FET described above (such as transconductance, $g_{m}$ ) is relatively unaffected due to the high capacitance of the polar layer. Consider the structure described in Fig. 1 to be composed of two capacitors in series. We have

$$
\frac{1}{C_{g}}=\frac{1}{C_{A}}+\frac{1}{C_{B}} \approx \frac{1}{C_{B}},
$$

where $C_{A}$ is the capacitance of either the polar or the insulator layer and $C_{B}$ is the capacitance of a similar FET structure without additional polar or insulator layer. This makes it 

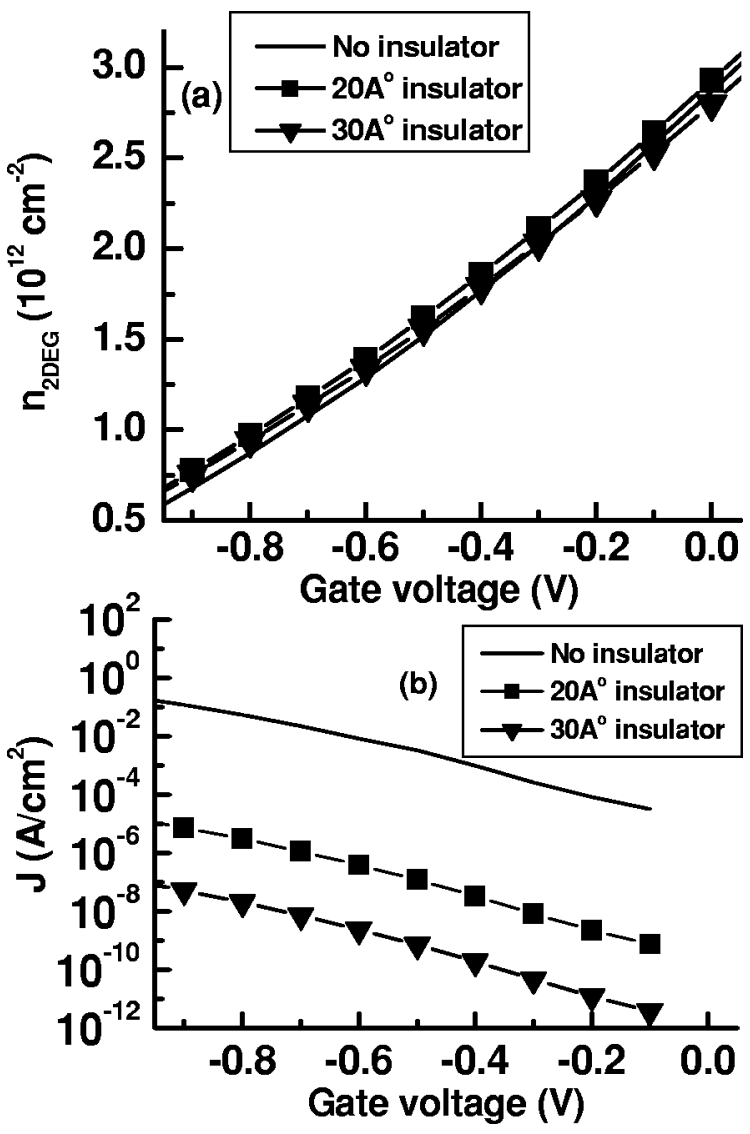

FIG. 3. $I-V$ characteristics and sheet charge densities, $n_{2 \mathrm{DEG}}$, for the device structure incorporating the insulator. The thickness of the $\mathrm{Al}_{0.3} \mathrm{Ga}_{0.7} \mathrm{~N}$ is taken to be $70 \AA$ A. Insulator parameters chosen: Dielectric constant $\kappa=29$, band gap, $E_{g}=3.74 \mathrm{eV}$, and Schottky barrier height, $\phi_{\mathrm{SB}}=1.15 \mathrm{eV}$.

amply clear that the introduction of the polar or insulator layer does not affect the transconductance, which is still adequately described by the relation,

$$
g_{m}=C_{g} v_{\text {sat }} \approx C_{B} v_{\text {sat }},
$$

which may be seen to be the same transconductance as that of a structure without either a polar or an insulator layer $\left(v_{\text {sat }}\right.$ is the saturation velocity of the carriers in the FET channel region). Since $g_{m}$ is the main figure of merit in description of FETs, it is apparent that the characteristics of circuits employing the proposed structure would not be affected much.

Next, we examine if a polar material can be exploited to fabricate low resistance ohmic contacts to a semiconductor structure. It is well known that in semiconductor technology, ${ }^{18}$ one way to convert a rectifying contact into an ohmic contact is to heavily dope the semiconductor in the region of the contact. Another way to achieve the same result is to use polar materials ${ }^{10}$ in the region of the contact. In the nitride system, the interfacial polar charge results from spontaneous polarization and piezoelectric effect, which cannot be altered once the crystal is grown. In ferroelectrics, such as $\mathrm{LiNbO}_{3}, \mathrm{SrTiO}_{3}$, etc., polarization values can be much larger than what is possible in nitrides and can also be controlled by external fields. Polar charge densities in the range of $10^{14} \mathrm{~cm}^{-2}$ are present in a number of ferroelectrics. For example, $\mathrm{LiNbO}_{3}$ has a polar charge of $\sim 2 \times 10^{14} \mathrm{~cm}^{-2}$ and
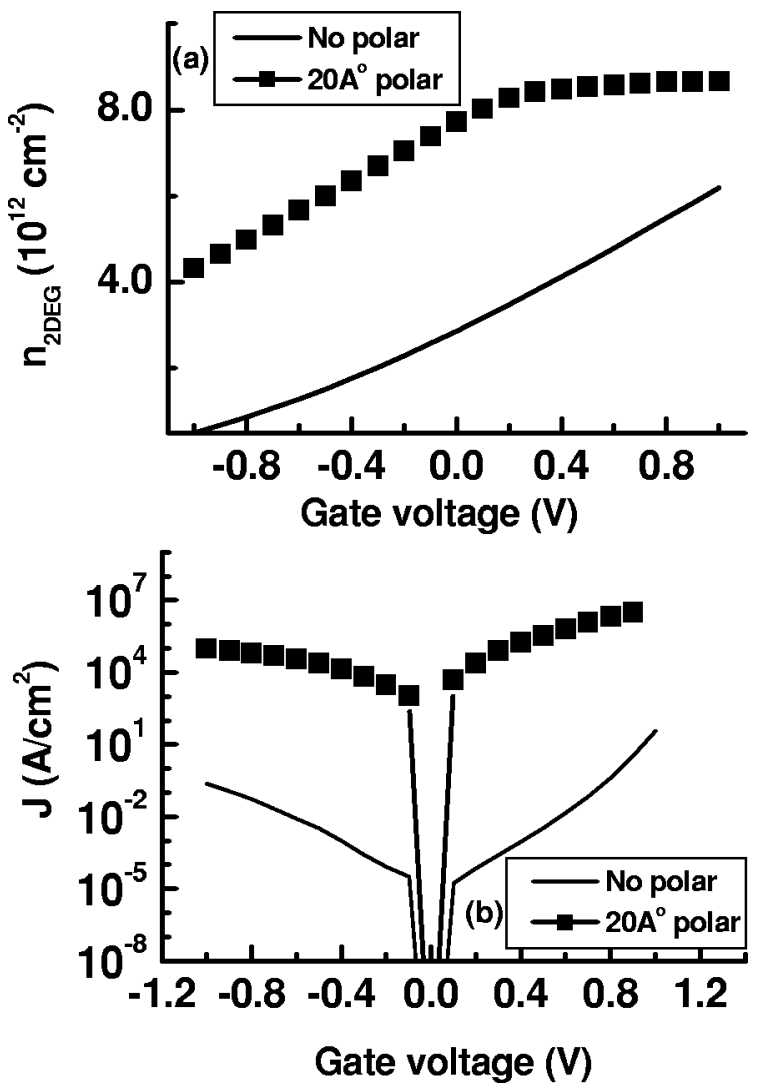

FIG. 4. $I-V$ characteristics and sheet charge densities, $n_{2 \mathrm{DEG}}$, for the device structure incorporating the polar material layer. The thickness of the $\mathrm{Al}_{0.3} \mathrm{Ga}_{0.7} \mathrm{~N}$ is taken to be $70 \AA$. Polar charge density at the interface between AlGaN and the polar material $=1 \times 10^{14} \mathrm{~cm}^{-2}$. Other parameters of the polar layer are taken to be the same as that of the insulator layer in Fig. 3.

has a very high coercive field. ${ }^{19}$ Ferroelectrics have been grown on nitrides and other semiconductors although their junction properties have not been reported. We examine the $I-V$ characteristics of a structure where a thin layer of a polar material with $\sigma=+10^{14} \mathrm{~cm}^{-2}$ is present between a metal and the $\mathrm{AlGaN} / \mathrm{GaN}$ structure. Results are shown in Fig. 4. As we can see, a very strong band bending can be induced which leads to a very high tunneling current. Figure 5 shows the band profiles and sheet charge distribution in the contact heterojunction in absence of "gate" bias. It shows that the barrier becomes very thin and the tunneling current density is increased by a factor of nearly $10^{5}$ (in either bias condition) when compared to the case without any polar layer. The use of the thin polar region thus changes the junction from rectifying to ohmic, without the need for heavy doping. High levels of doping are difficult to obtain in large band-gap materials. ${ }^{24-27}$

We also examine the influence of thickness, $d_{A}$, of the polar material. As can be seen in Fig. 6, an increase in the thickness of the polar material initially increases the tunneling current and the 2DEG sheet charge density. It can be seen that the positive polar charges at the $\mathrm{AlGaN} /$ polar material heterointerface lead to higher band bending when $d_{A}$ increases from $0 \AA$ to $20 \AA$. However, when the thickness $d_{A}$ is over $20 \AA$, the induced 2DEG charge at the AlGaN/polar material heterointerface starts to annul the effects of the band 


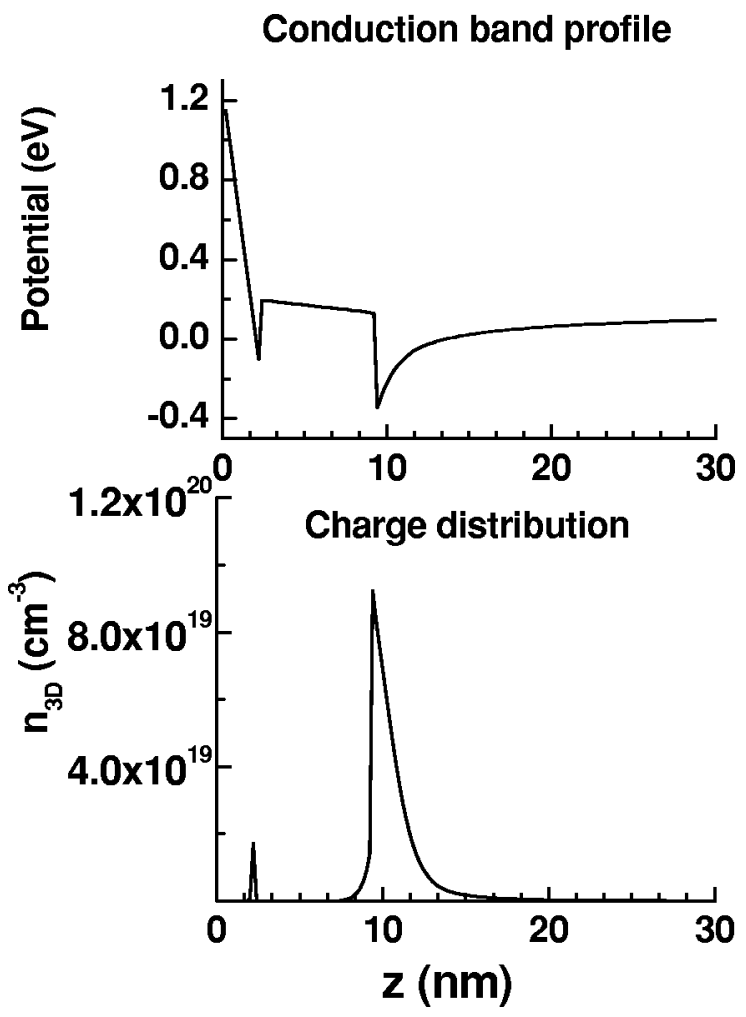

FIG. 5. Conduction-band profile and charge density distribution, $n_{3 \mathrm{D}}$, for the structure incorporating a polar material layer of thickness $20 \AA$ A. Gate voltage, $V_{g}=0 \mathrm{~V}$.

bending. Then, the barrier thickness, $d_{A}+d_{B}$, dominates the tunneling probability. Hence, there exists an optimal thickness $(\simeq 20 \AA)$ that corresponds to the highest tunneling current.

A study of the variation of AlGaN thickness with fixed polar material thickness indicates that while the charge density induced in the channel remains practically unchanged, the tunneling current exhibits an expected decrease with increase in that thickness.

\section{CONCLUSIONS}

In this work, we developed a drift-diffusion charge control model and examined vertical transport in GaN/AlGaN, GaN/AlGaN/insulator, and GaN/AlGaN/polar material layer heterostructure junctions.

The introduction of the high- $\kappa$ insulator layer shows that we can reduce the tunneling current density without affecting the sheet charge density or changing the circuit characteristics of the device significantly. This is of use in designing low gate leakage structures in the gate region of HEMTs without affecting sheet charge density or transconductance [see Eq. (13)] in the channel region.

We have examined the use of a strongly polar layer, which forms ohmic junctions in the barrier region. We have also found that there exists a certain optimal thickness of the polar material that yields the highest possible tunneling current. This is likely to be of immense use in the design of low resistance contacts. Since, the chosen value of the dielectric constant, $\kappa=29$, is quite low, we do not expect the delays in
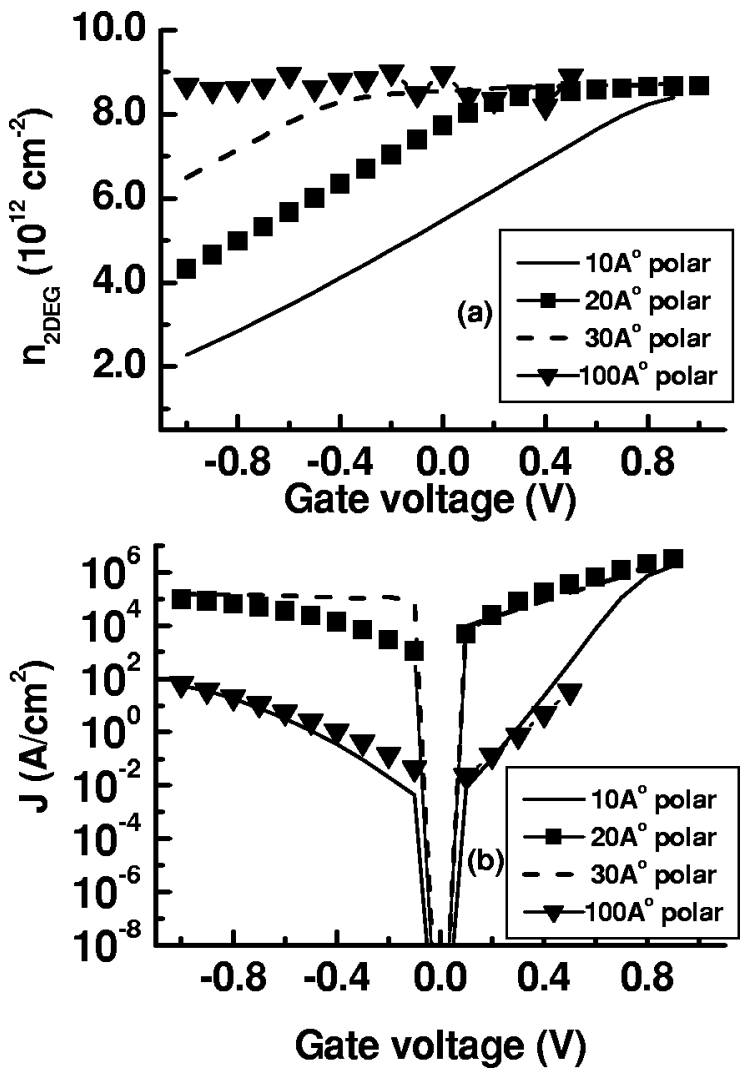

FIG. 6. Dependence of $I-V$ characteristics and sheet charge densities, $n_{2 \mathrm{DEG}}$, on thickness, $d_{A}$, of the polar layer.

the contact to be increased unduly due to capacitive linkage. Further, the direction of the polarization in the polar material can be changed at the growth time by using poling. This can be used to form self-insulating regions and suggests a growth method by using laterally varying electric fields during crystal growth.

By using a combination of polar materials and high dielectric constant insulators, it should be possible to design structures by adding an insulator at gate to reduce the leakage current and use polar layer at drain and source to form ohmic contacts without heavy doping. Future work would involve simultaneous optimization of tunneling current by varying $\mathrm{AlGaN}$ as well as polar material thickness.

\section{ACKNOWLEDGMENT}

This work was supported by Grant No. F004815 from the U.S. Office of Naval Research.

${ }^{1}$ O. Aktas, Z. F. Fan, A. Botchkarev, S. N. Mohammad, M. Roth, T. Jenkins, L. Kehias, and H. Morkoc, IEEE Electron Device Lett. 18, 293 (1997).

${ }^{2}$ M. S. Shur and M. A. Khan, MRS Bull. 22, 44 (1997).

${ }^{3}$ Y. F. Wu, S. Keller, P. Kozodoy, B. P. Keller, P. Parikh, D. Kapolnek, S. P. DenBaars, and U. K. Mishra, IEEE Electron Device Lett. 18, 290 (1997).

${ }^{4}$ U. K. Mishra, Y. F. Wu, B. P. Keller, S. Keller, and S. P. DenBaars, IEEE Trans. Microwave Theory Tech. 46, 756 (1998).

${ }^{5}$ R. Dimitrov, L. Wittmer, H. Felsl, A. Mitchell, O. Ambacher, and M. Stutzmann, Phys. Status Solidi A 168, R7 (1998).

${ }^{6}$ F. Bernardini, V. Fiorentini, and D. Vanderbilt, Phys. Rev. B 56, 10024 (1997).

${ }^{7}$ O. Ambacher, J. Smart, J. R. Shealy, N. G. Weimann, K. Chu, M. Murphy, W. J. Schaff, L. F. Eastman, R. Dimitrov, L. Wittmer, M. Stutzmann, W. 
Rieger, and J. Hilsenbeck, J. Appl. Phys. 85, 3222 (1999).

${ }^{8}$ Y. Zhang, I. P. Smorchkova, C. R. Elsass, S. Keller, J. P. Ibbetson, S. Denbaars, U. Mishra, and J. Singh, J. Appl. Phys. 87, 7981 (2000).

${ }^{9}$ N. Maeda, T. Saitoh, K. Tsubaki, T. Nishida, and N. Kobayashi, Appl. Phys. Lett. 76, 3118 (2000).

${ }^{10}$ M. Singh, Y. Zhang, J. Singh, and U. Mishra, Appl. Phys. Lett. 77, 1867 (2000).

${ }^{11}$ M. Singh, J. Singh, and U. Mishra, J. Appl. Phys. 91, 2989 (2002).

${ }^{12}$ M. Singh, Y.-R. Wu, and J. Singh, Solid-State Electron. (to be published).

${ }^{13}$ H. K. Gummel, IEEE Trans. Electron Devices 11, 455 (1964).

${ }^{14}$ K. Horio and H. Yanai, IEEE Trans. Electron Devices 37, 1093 (1990).

${ }^{15}$ K. Yang, J. R. East, and G. I. Haddad, Solid-State Electron. 36, 321 (1993).

${ }^{16}$ Y. Zhang and J. Singh, J. Appl. Phys. 85, 587 (1999).

${ }^{17} \mathrm{~J}$. Singh, Physics of Semiconductors and Their Heterostructures (McGraw-Hill, New York, 1993).

${ }^{18}$ S. M. Sze, Semiconductor Devices Physics and Technology (Wiley, New York, 1985).
${ }^{19}$ S. Kim, V. Gopalan, and A. Gruverman, Appl. Phys. Lett. 80, 2740 (2002).

${ }^{20}$ A. G. Zembilgotov, N. A. Pertsev, H. Kohlstedt, and R. Waser, J. Appl. Phys. 91, 2247 (2002).

${ }^{21}$ R. Droopad, Z. Yu, J. Ramdani, L. Hilt, J. C. Overgaard, J. L. Edwards, J. Finder, K. Eisenbeiser, J. Wang, V. Kaushik, B.-Y. Nguyen, and B. Ooms, J. Cryst. Growth 227, 936 (2001).

${ }^{22}$ R. Droopad, Z. Yu, J. Ramdani, L. Hilt, J. Curless, C. Overgaard, J. L. Edwards, Jr., J. Finder, K. Eisenbeiser, and W. Ooms, Mater. Sci. Eng., B 87, 292 (2001).

${ }^{23}$ P. Hansen, Y. Wu, L. Shen, S. Heikman, S. P. DenBaars, R. A. York, U. K. Mishra, and J. S. Speck, Center for Advanced Nitride Electronics CANE/ MURI REVIEW (University of California, Santa Barbara, 2003).

${ }^{24}$ P. Kozodoy, H. Xing, S. P. DenBaars, U. K. Mishra, A. Saxler, R. Perrin, S. Elhamri, and W. C. Mitchel, J. Appl. Phys. 87, 1832 (2000).

${ }^{25}$ S. B. Zhang, S. H. Wei, and A. Zunger, Phys. Rev. Lett. 84, 1232 (2000).

${ }^{26}$ W. Walukiewicz, Physica B 302, 123 (2001).

${ }^{27}$ R. Y. Korotkov, J. M. Gregie, and B. W. Wessels, Opto-Elec. Rev. 10, 243 (2002). 